persons with COPD is no different than in those without COPD

(e) Amoxicillin would be a reasonable antibiotic choice if the patient is relatively well (ie he has non-severe CAP)

10 A 44-year-old man is admitted to hospital with a five-day history of fever, confusion and a dry cough. His respiratory rate is 35 per min and blood pressure $100 / 55 \mathrm{mmHg}$ on admission. CXR is consistent with a diagnosis of CAP. Which of the following statements are correct and which false?

(a) A history of travel to Ibiza makes Legionella pneumonia more likely

(b) A background of rheumatoid arthritis is a specific risk factor for Staphylococcus aureus pneumonia

(c) His estimated risk of mortality is higher than $15 \%$

(d) Intravenous cefuroxime in combination with clarithromycin would be a reasonable choice of antibiotics

(e) An initial C-reactive protein of over $300 \mathrm{mg} / \mathrm{l}$ is a poor prognostic feature
Guidelines on completing the answer sheet for those who wish to
submit their answers

A loose leaf answer sheet is enclosed, which will be marked electronically at the Royal College of Physicians. Answer sheets must be returned by 21 July 2004 to: CME Department (SAQs), Royal College of Physicians, 11 St Andrews Place, London NW1 4LE.

Overseas members only can fax their answers to 02074874156

Correct answers will be published in the next issue of Clinical Medicine.

*Further details on CME are available from the CME department at the Royal College of

Physicians (address above or telephone 02079351174 extension 306 or 309).

Your completed answer sheet will be scanned to enable a quick and accurate analysis of results. To aid this process, please keep the following in mind:

1 Please print your GMC Number firmly and neatly

2 Only write in allocated areas on the form

3 Only use pens with black or dark blue ink

4 For optimum accuracy, ensure printed numbers avoid contact with box edges

5 Please shade circles like this: Not like this: $\varnothing$

6 Please mark any mistakes made like this: $\not \gamma$

7 Please do not mark any of the black squares on the corners of each page

8 Please fill in your full name and address on the back of the answer sheet in the space provided; this will be used to mail the form back to you after marking.

\title{
CME Neurology SAQs
}

\section{Answers to the CME SAQs published in Clinical Medicine March/April 2004}

$\begin{array}{llllllllll}\text { Q1 } & \text { Q2 } & \text { Q3 } & \text { Q4 } & \text { Q5 } & \text { Q6 } & \text { Q7 } & \text { Q8 } & \text { Q9 } & \text { Q10 } \\ \text { a) } F & \text { a) } F & \text { a) } T & \text { a) } F & \text { a) } F & \text { a) } F & \text { a) } F & \text { a) } F & \text { a) } F & \text { a) } F \\ \text { b) } F & \text { b) } T & \text { b) } F & \text { b) } F & \text { b) } T & \text { b) } T & \text { b) } F & \text { b) } F & \text { b) } F & \text { b) } F \\ \text { c) } F & \text { c) } F & \text { c) } F & \text { c) } F & \text { c) } F & \text { c) } T & \text { c) } T & \text { c) } T & \text { c) } F & \text { c) } T \\ \text { d) } F & \text { d) } F & \text { d) } T & \text { d) } F & \text { d) } F & \text { d) } F & \text { d) } F & \text { d) } T & \text { d) } T & \text { d) } T \\ \text { e) } T & \text { e) } F & \text { e) } F & \text { e) } T & \text { e) } F & \text { e) } F & \text { e) } F & \text { e) } F & \text { e) } F & \text { e) } F\end{array}$

